\title{
OMALIZUMAB MAY FACILITATE DRUG DESENSITIZATION IN PATIENTS FAILING STANDARD PROTOCOLS
}

\author{
Hatice Betul Gemici Karaaslan ${ }^{1}$, Esra Karabag ${ }^{2}$, Ruveyda Gulmez ${ }^{2}$, Nur Canpolat ${ }^{2}$, Ayca \\ Kiykim $^{1}$, and Haluk Cokugras ${ }^{2}$ \\ ${ }^{1}$ Istanbul Universitesi-Cerrahpasa \\ ${ }^{2}$ Istanbul University-Cerrahpasa Cerrahpasa Faculty of Medicine
}

March 8, 2022

\section{OMALIZUMAB MAY FACILITATE DRUG DESENSITIZATION IN PATIENTS FAILING STANDARD PROTOCOLS}

To the Editor,

Nephrotic syndrome (NS) is a common glomerular disorder in children, for which steroids are the firstline treatment. While most children with NS respond to steroid therapy, $20 \%$ of children are resistant to steroids. Some children with steroid-responsive NS develop a frequently relapsing or steroid-dependent course and experience significant side effects of steroid therapy. Alternative medications such as calcineurin inhibitors, mycophenolate mofetil, cyclophosphamide, and rituximab, an anti-CD20 monoclonal antibody, are being considered for such patients with difficult-to-treat NS $(1,2)$.

Hypersensitivity reactions to monoclonal antibodies are quite limited in clinical practice, such as the release of cytokines that occur during intravenous infusion. However, IgE-mediated reactions may also occur. Life-threatening IgE-mediated reactions such as anaphylaxis lead to discontinuation of treatment or conversion to a less beneficial treatment. Rapid drug desensitization (RDD) is a therapeutic option that allows continuation of treatment with the causative drug (3). Omalizumab is a recombinant humanized anti-IgE monoclonal antibody. Treatment indications include severe asthma and idiopathic chronic urticaria. However, the efficacy of omalizumab has also been described in food allergy, as a bridge to oral immunotherapies, atopic dermatitis, idiopathic anaphylaxis, and mastocytosis (4). Previous studies have reported the use of omalizumab for rapid desensitization to chemotherapeutic agents (5). Here, we describe a patient with steroid-resistant NS who developed anaphylaxis on the first infusion of rituximab and subsequent type 1 hypersensitivity reactions during desensitization trials with rituximab using 12-, 16-, and 20-step protocols.

A 4-year-old boy diagnosed with steroid-resistant NS and unresponsive to calcineurin inhibitors, either cyclosporine A or tacrolimus, and in their combination with mycophenolate mofetil, received rituximab. After premedication with methylprednisolone at a dose of $2 \mathrm{mg} / \mathrm{kg}$, rituximab $375 \mathrm{mg} / \mathrm{m}^{2}$ was administered intravenously. During the infusion, he developed anaphylaxis (vomiting and dyspnea). The infusion was stopped and intramuscular epinephrine was administered, and a 12-step rapid desensitization protocol was planned for further infusions (Table 1). He was premedicated with H1 blockers and systemic steroids. However, the patient developed breakthrough reactions (urticaria) that required additional antihistamines at the 4th step. Infusion was resumed at a slower rate. Ten minutes after re-administration, generalized urticaria and angioedema developed. Montelukast was administered according to ENDA/EAACI recommendations for rapid desensitization in drug allergy (6). After 7 days, the protocol was modified to administer 375 $\mathrm{mg} / \mathrm{m}^{2}$ rituximab in 16 steps with premedication (Table 2). The patient developed generalized urticaria and angioedema again at the 4th step. The next week, the desensitization protocol was designed with 375 
$\mathrm{mg} / \mathrm{m}^{2}$ rituximab in 20 steps (Table 3). Again, generalized urticaria and angioedema occurred in the 2nd step. A skin prick test was performed 3 weeks after the initial reaction. During the skin prick test, the patient developed generalized urticaria.

In the absence of alternative treatment options for NS, desensitization with omalizumab treatment was suggested to prevent hypersensitivity reactions to rituksimab. Omalizumab (patient weight: $17.5 \mathrm{~kg}$; total IgE:71 UI /ml; dose: $150 \mathrm{mg} /$ every 2 weeks) was added to treatment. The last omalizumab dose was administered 1 day before the following desensitization. After premedication, $375 \mathrm{mg} / \mathrm{m}^{2}$ rituximab was administered in 20 steps. In the 5 th step, the patient developed local urticaria requiring an antihistamine. The infusion was resumed and successfully completed. Under omalizumab treatment, the patient was administered $375 \mathrm{mg} / \mathrm{m}^{2}$ rituximab for the second time in a 20-step protocol. After the 6th dose, the interval of omalizumab treatment was changed to every 4 weeks. The rituximab dose was then increased to $750 \mathrm{mg} / \mathrm{m}^{2}$ at the third infusion. After premedication, the 20-step desensitization protocols were successfully applied in the following days.

Rituximab is a chimeric monoclonal antibody that targets the CD20 antigen on the surface of B cells and causes elimination of B lymphocytes by complement- and antibody-dependent cellular cytotoxicity for 6-12 months (2). Efficacy has also been demonstrated in the treatment of steroid-resistant NS (7). Rituximab is one of the most common biologic agents with infusion-related reactions. Rituximab-associated hypersensitivity reactions can be classified as infusion-related reactions, cytokine release, IgE-mediated/nonIgE-mediated hypersensitivity reactions, mixed reactions, type 3 and type 4 hypersensitivity reactions (8).

Omalizumab is a recombinant humanized IgG1 monoclonal antibody and prevents degranulation in effector cells by specifically binding to the FceRI receptor site of free IgE, causing a decrease in the level of free $\operatorname{IgE}$ in serum, and causing downregulation of FceRI receptors (9). Omalizumab has previously been used as a co-adjuvant in RDD along with insulin, the enzyme elosulfase alpha, chemotherapeutic agents, and aspirin (9). In a randomized, double-blind, placebo-controlled trial by Lang et al, desensitization was achieved after 16 weeks treatment with omalizumab. In other case reports, omalizumab treatment was generally started 7-14 days before RDD; only in one case report was the first omalizumab dose given 4 days before RDD (9). In previous studies, omalizumab dosing in allergic asthma was based on patient weight and total IgE. In our case, we administered 6 doses of omalizumab (150 mg/dose) every two weeks before desensitization and continued treatment once a month until completion of rituximab therapy. In patients with successful RDD on omalizumab, RDD steps can be reduced on subsequent infusions. Arroaberran et al. (10) presented a patient who tolerated elosulfase alpha enzyme without desensitization after omalizumab treatment.

In conclusion, omalizumab may facilitate desensitization protocols and allow continuation of the preferred treatment.

${ }^{1}$ Hatice Betul Gemici Karaaslan,

betulgmc@gmail.com

${ }^{2}$ Esra Karabag Yilmaz,

esrakarabag.dr@hotmail.com

${ }^{2}$ Ruveyda Gulmez, akerruveyda@gmail.com

${ }^{2}$ Nur Canpolat, ncanpolat2000@hotmail.com

${ }^{1}$ Ayca Kiykim, ayca.kiykim@iuc.edu.tr

${ }^{1}$ Haluk Cezmi Cokugras, cokugras@gmail.com

${ }^{1}$ Istanbul University-Cerrahpasa, Department of Pediatric Immunology and Allergy, Istanbul

${ }^{2}$ Istanbul University-Cerrahpasa, Department of Pediatric Nephrology, Istanbul 
1. Rovin BH, Adler SG, Barratt J et al (2021) Executive summary of the KDIGO 2021 Guideline for the Management of Glomerular Diseases. Kidney Int 100:753-779.

2. Ataca P, Atilla E, Kendir R, Bavbek S, Ozcan M. Successful desensitization of a patient with rituximab hypersensitivity. Case Reports Immunol. 2015;2015:524507.

3. D. I. Hong, L. Bankova, K. N. Cahill, T. Kyin, and M. C. Castells. Allergy to monoclonal antibodies: cutting-edge desensitization methods for cutting-edge therapies. Expert Review of Clinical Immunology, vol. 8, no. 1, pp. 43-54, 2012.

4. Pichler WJ, Hausmann O. Classification of drug hypersensitivity into allergic, p-i, and pseudo-allergic forms. Int Arch Allergy Immunol 2016; 171:166 - 179.

5. Bumbacea RS, Ali S, Corcea SL, Spiru L, Nitipir C, Strambu V, Bumbacea D. Omalizumab for successful chemotherapy desensitisation: What we know so far. Clin Transl Allergy. 2021 Dec 13;11(10):e12086.

6. Taha R, El-Haddad H, Almuallim A, Alshaiki F, Obaid E, Almoallim H. Systematic review of the role of rituximab in treatment of antineutrophil cytoplasmic autoantibody-associated vasculitis, hepatitis C virus-related cryoglobulinemic vasculitis, Henoch-Schonlein purpura, ankylosing spondylitis, and Raynaud's phenomenon. Open Access Rheumatol 2017;9:201-14.

7. Görgülü B, Seval GC, Kendirlinan R, Toprak SK, Özcan M, Bavbek S. Rapid Drug Desensitization With Rituximab in 24 Cases: A Single-Center Experience. J Investig Allergol Clin Immunol. 2019;29(6):468-470.

8. Isabwe GAC, Neuer MG, de las Vecillas Sanchez L, Lynch DM, Marquis K, Castells M. Hypersensitivity reactions to therapeutic monoclonal antibodies: phenotypes and endotypes. J Allergy Clin Immunol. 2018;142(1):159-71

9. Fernandez J, Ruano-Zaragoza M, Blanca-Lopez N. Omalizumab and other biologics in drug desensitization. Curr Opin Allergy Clin Immunol. 2020 Aug;20(4):333-337.

10. Arroabarren E, Aznal E, Anda M, Sanchez-Valverde F. Anaphylaxis after Elosulfase A infusion: Omalizumab as coadjuvant for enzyme replacement therapy desensitization. Pediatr Allergy Immunol. 2019 Jun;30(4):491-494.

\begin{tabular}{lllllll}
\hline Step & Solution & Rate $(\mathrm{mL} / \mathrm{hr})$ & $\begin{array}{l}\text { Time per } \\
\text { step }\end{array}$ & $\begin{array}{l}\text { Volume per } \\
\text { step }(\mathbf{m L})\end{array}$ & $\begin{array}{l}\text { Dose/Step } \\
(\mathrm{mg})\end{array}$ & $\begin{array}{l}\text { Cumulative } \\
\text { Dose }(\mathbf{m g})\end{array}$ \\
\hline 1 & $\mathrm{~A}$ & 2 & $15 \mathrm{~min}$ & 0.5 & 0.006 & 0.006 \\
2 & $\mathrm{~A}$ & 5 & $15 \mathrm{~min}$ & 1.25 & 0.015 & 0.021 \\
3 & $\mathrm{~A}$ & $15 \mathrm{~min}$ & 2.5 & 0.03 & 0.051 \\
4 & $\mathrm{~A}$ & 10 & $15 \mathrm{~min}$ & 5 & 0.06 & 0.111 \\
5 & $\mathrm{~B}$ & 5 & $15 \mathrm{~min}$ & 1.25 & 0.125 & 0.236 \\
6 & $\mathrm{~B}$ & 10 & $15 \mathrm{~min}$ & 2.5 & 0.25 & 0.486 \\
7 & $\mathrm{~B}$ & $15 \mathrm{~min}$ & 5 & 0.5 & 0.986 \\
8 & $\mathrm{~B}$ & $15 \mathrm{~min}$ & 10 & 1 & 1.986 \\
9 & $\mathrm{C}$ & $15 \mathrm{~min}$ & 2.5 & 2.65 & 4.636 \\
10 & $\mathrm{C}$ & 20 & $15 \mathrm{~min}$ & 5 & 5.3 & 9.936 \\
11 & $\mathrm{C}$ & 40 & $15 \mathrm{~min}$ & 10 & 10.6 & 20.536 \\
12 & $\mathrm{C}$ & 80 & $173 \mathrm{~min}$ & 230 & 244.464 & 265 \\
\hline
\end{tabular}

Table 1. Rituximab desensitization protocol for 12 steps $\left(375 \mathrm{mg} / \mathrm{m}^{2}\right)$

Solution A: $250 \mathrm{~mL}, 5 \%$ dextrose, $(0.012 \mathrm{mg} / \mathrm{mL})$.

Solution B: $250 \mathrm{~mL}, 5 \%$ dextrose, $(0.106 \mathrm{mg} / \mathrm{mL})$.

Solution C: $250 \mathrm{~mL}, 5 \%$ dextrose, $(1.06 \mathrm{mg} / \mathrm{mL})$. 


\begin{tabular}{|c|c|c|c|c|c|c|}
\hline Step & Solution & $\begin{array}{l}\text { Rate } \\
\text { (mL/hour) }\end{array}$ & $\begin{array}{l}\text { Time per } \\
\text { step }\end{array}$ & $\begin{array}{l}\text { Volume } \\
\text { per step } \\
(\mathrm{mL})\end{array}$ & $\begin{array}{l}\text { Dose/Step } \\
\text { (mg) }\end{array}$ & $\begin{array}{l}\text { Cumulative } \\
\text { Dose (mg) }\end{array}$ \\
\hline 1 & $\mathrm{~A}$ & 0.5 & $15 \mathrm{~min}$ & 0.125 & 0.00015 & 0.00015 \\
\hline 2 & A & 1 & 15 min & 0.25 & 0.0003 & 0.00045 \\
\hline 3 & $\mathrm{~A}$ & 2 & $15 \mathrm{~min}$ & 0.5 & 0.0006 & 0.00105 \\
\hline 4 & A & 4 & $15 \mathrm{~min}$ & 1 & 0.0012 & 0.00225 \\
\hline 5 & B & 1 & $15 \mathrm{~min}$ & 0.25 & 0.003 & 0.00525 \\
\hline 6 & B & 2 & $15 \mathrm{~min}$ & 0.5 & 0.006 & 0.01125 \\
\hline 7 & B & 4 & $15 \mathrm{~min}$ & 1 & 0.012 & 0.02325 \\
\hline 8 & B & 8 & $15 \mathrm{~min}$ & 2 & 0.024 & 0.04725 \\
\hline 9 & $\mathrm{C}$ & 2 & $15 \mathrm{~min}$ & 0.5 & 0.053 & 0.10025 \\
\hline 10 & $\mathrm{C}$ & 4 & $15 \mathrm{~min}$ & 1 & 0.106 & 0.20625 \\
\hline 11 & $\mathrm{C}$ & 8 & $15 \min$ & 2 & 0.212 & 0.41825 \\
\hline 12 & $\mathrm{C}$ & 16 & $15 \min$ & 4 & 0.424 & 0.84225 \\
\hline 13 & $\mathrm{D}$ & 4 & $15 \min$ & 1 & 1.06 & 1.90225 \\
\hline 14 & $\mathrm{D}$ & 10 & $15 \min$ & 2.5 & 2.65 & 4.55225 \\
\hline 15 & $\mathrm{D}$ & 20 & $15 \mathrm{~min}$ & 5 & 5.3 & 9.85225 \\
\hline 16 & $\mathrm{D}$ & 40 & $361 \min$ & 240.7 & 255.14 & 265 \\
\hline
\end{tabular}

Table 2. Rituximab desensitization protocol for 16 steps $\left(375 \mathrm{mg} / \mathrm{m}^{2}\right)$

Solution A: $250 \mathrm{~mL}, 5 \%$ dextrose, $(0.0012 \mathrm{mg} / \mathrm{mL})$.

Solution B: $250 \mathrm{~mL}, 5 \%$ dextrose, $(0.012 \mathrm{mg} / \mathrm{mL})$.

Solution C: $250 \mathrm{~mL}, 5 \%$ dextrose, $(0.106 \mathrm{mg} / \mathrm{mL})$.

Solution D: $250 \mathrm{~mL}, 5 \%$ dextrose, $(1.06 \mathrm{mg} / \mathrm{mL})$.

\begin{tabular}{|c|c|c|c|c|c|c|}
\hline Step & Solution & $\begin{array}{l}\text { Rate } \\
\text { (mL/hour) }\end{array}$ & $\begin{array}{l}\text { Time per } \\
\text { step }\end{array}$ & $\begin{array}{l}\text { Volume } \\
\text { per step } \\
(\mathrm{mL})\end{array}$ & $\begin{array}{l}\text { Dose/Step } \\
(\mathrm{mg})\end{array}$ & $\begin{array}{l}\text { Cumulative } \\
\text { Dose (mg) }\end{array}$ \\
\hline 1 & $\mathrm{~A}$ & 0.1 & $15 \mathrm{~min}$ & 0.025 & 0.000003 & 0.000003 \\
\hline 2 & $\mathrm{~A}$ & 0.2 & $15 \mathrm{~min}$ & 0.05 & 0.000006 & 0.000009 \\
\hline 3 & $\mathrm{~A}$ & 0.5 & $15 \mathrm{~min}$ & 0.125 & 0.000015 & 0.000024 \\
\hline 4 & $\mathrm{~A}$ & 1 & $15 \mathrm{~min}$ & 0.25 & 0.00003 & 0.000054 \\
\hline 5 & B & 0.5 & $15 \mathrm{~min}$ & 0.125 & 0.00015 & 0.000204 \\
\hline 6 & B & 1 & $15 \mathrm{~min}$ & 0.25 & 0.0003 & 0.000504 \\
\hline 7 & B & 2 & $15 \mathrm{~min}$ & 0.5 & 0.0006 & 0.001104 \\
\hline 8 & B & 4 & $15 \min$ & 1 & 0.0012 & 0.002304 \\
\hline 9 & $\mathrm{C}$ & 1 & $15 \mathrm{~min}$ & 0.25 & 0.003 & 0.005304 \\
\hline 10 & $\mathrm{C}$ & 2 & $15 \mathrm{~min}$ & 0.5 & 0.006 & 0.011304 \\
\hline 11 & $\mathrm{C}$ & 4 & $15 \min$ & 1 & 0.012 & 0.023304 \\
\hline 12 & $\mathrm{C}$ & 8 & $15 \min$ & 2 & 0.024 & 0.047304 \\
\hline 13 & $\mathrm{D}$ & 2 & $15 \mathrm{~min}$ & 0.5 & 0.053 & 0.100304 \\
\hline 14 & $\mathrm{D}$ & 4 & $15 \mathrm{~min}$ & 1 & 0.106 & 0.206304 \\
\hline 15 & $\mathrm{D}$ & 8 & $15 \mathrm{~min}$ & 2 & 0.212 & 0.418304 \\
\hline 16 & $\mathrm{D}$ & 16 & $15 \mathrm{~min}$ & 4 & 0.424 & 0.842304 \\
\hline 17 & $\mathrm{E}$ & 4 & $15 \mathrm{~min}$ & 1 & 1.06 & 1.902304 \\
\hline 18 & $\mathrm{E}$ & 10 & $15 \min$ & 2.5 & 2.65 & 4.5523049 \\
\hline
\end{tabular}




\begin{tabular}{|c|c|c|c|c|c|c|}
\hline Step & Solution & $\begin{array}{l}\text { Rate } \\
\text { (mL/hour) }\end{array}$ & $\begin{array}{l}\text { Time per } \\
\text { step }\end{array}$ & $\begin{array}{l}\text { Volume } \\
\text { per step } \\
(\mathrm{mL})\end{array}$ & $\begin{array}{l}\text { Dose/Step } \\
\text { (mg) }\end{array}$ & $\begin{array}{l}\text { Cumulative } \\
\text { Dose (mg) }\end{array}$ \\
\hline 19 & $\mathrm{E}$ & 20 & $15 \mathrm{~min}$ & 5 & 5.3 & 15.152 \\
\hline 20 & $\mathrm{E}$ & 40 & $312 \min$ & 208 & 249.8 & 265 \\
\hline
\end{tabular}

Table 3. Rituximab desensitization protocol for 20 steps $\left(375 \mathrm{mg} / \mathrm{m}^{2}\right)$

Solution A: $250 \mathrm{~mL}, 5 \%$ dextrose, $(0.00012 \mathrm{mg} / \mathrm{mL})$.

Solution B: $250 \mathrm{~mL}, 5 \%$ dextrose, $(0.0012 \mathrm{mg} / \mathrm{mL})$.

Solution C: $250 \mathrm{~mL}, 5 \%$ dextrose, $(0.012 \mathrm{mg} / \mathrm{mL})$.

Solution D: $250 \mathrm{~mL}, 5 \%$ dextrose, $(0.106 \mathrm{mg} / \mathrm{mL})$.

Solution E: $250 \mathrm{~mL}, 5 \%$ dextrose, $(1.06 \mathrm{mg} / \mathrm{mL})$. 